\title{
Psychological Interventions for Recurrence Prevention in Adolescent Depression: A Systematic Review
}

\author{
Chonnakarn Jatchavala, M.D. ${ }^{1}$, Stella Chan, DPhil, DClinPsy, CPsychol, FBPsS ${ }^{2}$ \\ 'Department of Psychiatry, Faculty of Medicine, Prince of Songkla University, Hat Yai, Songkhla 90110, Thailand. \\ ${ }^{2}$ School of Health in Social Science, University of Edinburgh, Doorway 6, Medical Quad, Teviot Place, Edinburgh EH8 9AG, \\ United Kingdom. \\ Received 15 November 2017 • Accepted 23 January 2018 • Published online 20 August 2018
}

\section{Abstract:}

Objective: To determine the effectiveness of psychological interventions in depressive adolescents to prevent recurrent depressive episodes.

Material and Methods: English databases, including Embase, Medline, Global Health, and PsychINFO, were searched for studies that examined the effectiveness of psychological interventions in preventing recurrent depression. Eight articles were found in the electronic databases and 4 studies were found from a manual search. Methodological quality was assessed using the Cochrane Collaboration's tool.

Results: One quasi-randomized control trial and 11 randomized control trials met the inclusion criteria. A systematic review of the 12 studies was conducted. The studies involved 1,641 participants that represented different effects of recurrence prevention interventions at $6,9,12,24$, and 33 months following treatment completion. Cognitive behavioral therapy (CBT) group intervention showed effectiveness in recurrence prevention in depressive adolescents in 6-33 months. Supportive psychotherapy and family therapy also prevented recurrent depression at 24-month follow-up, while psychodynamic/psychoanalytic psychotherapy and interpersonal psychotherapy could prevent recurrence in depressive adolescents for 12 months following completion of the intervention.

Conclusion: Among the psychological interventions studied in the included articles, CBT group intervention showed the greatest long-term effect since group CBT could prevent recurrent depression in adolescents up to 33 months.

Keywords: adolescent, depression, psychological intervention, recurrence prevention

Contact: Chonnakarn Jatchavala, M.D.

Department of Psychiatry, Faculty of Medicine, Prince of Songkla University,

Hat Yai, Songkhla 90110, Thailand.

E-mail: jchonnak@gmail.com
J Health Sci Med Res 2018;36(3):171-183 DOI: http://dx.doi.org/10.31584/jhsmr.2018.36.3.15 www.jhsmr.org 


\section{Introduction}

According to the World Health Organization, ${ }^{1}$ approximately $20.0 \%$ of adolescents around the world reported having mental health difficulties with depression being one of the most common problems. Clinical depression among adolescents was stated to have high prevalence rates: $6.0 \%$ at the age of 15 years old which increased to $17.4 \%$ at the age of 18 years old. ${ }^{2}$ Depression leads to significantly increased risks of physical diseases, relationship difficulties, and cognitive problems, especially in college students. ${ }^{3}$

Many studies have suggested that depression in adolescence is associated with long-term negative consequences, for instance increased risks of later depression, anxiety, and suicidal behaviors. ${ }^{4}$ In addition, depression itself is a highly recurrent disorder in which the subsequent episodes will usually begin within five years following the first episode of depression. Onset during adolescence is one of the predictors of continued risk for recurrences of depressive episodes and several psychosocial comorbidities. $^{5}$

Because of the high prevalence and serious consequences of adolescent depression, research into evaluating the effectiveness of both depression prevention programs and therapeutic interventions is of great importance. ${ }^{6}$ However, the research examining prevention strategies for depressive adolescents or recurrence prevention interventions did not clearly identify which ones were the most effective. Most research aimed to study the efficacy of antidepressants or other pharmacological interventions, not psychosocial intervention. ${ }^{7}$

Psychological intervention for prevention was divided into universal, selective, and indicated. ${ }^{8}$ Universal prevention embraces the entire population, whereas selective prevention includes adolescents with particular psychopathological risk factors for depression, while indicated prevention involves those with subclinical or mild- to-moderate depressive symptoms. Relapse prevention should be categorized as an indicated prevention in terms of incomplete remission but it should be a selective prevention in patients with complete remission. ${ }^{9}$

A number of meta-analyses found that at 6-month follow-up, selective and indicated prevention programs were more effective than universal programs. ${ }^{10}$ Even though Stice and colleagues found that selective prevention programs yielded larger effects than universal ones, universal programs were still considered useful due to their cost-effectiveness and public health promotion. ${ }^{11}$

Ishikawa and co-authors reported the effect on depressive symptoms of classroom-based social skills training as an example of preventive intervention which was theoretically maintained for 1 year. ${ }^{12}$ But in terms of the longer effects of classroom-based interventions, Sato and colleagues indicated that coping strategy training was more efficient in elementary school students and early adolescents who were approximately 9-12 years old, due to their cognitive abilities. ${ }^{9}$

Current studies revealed that most of the evidencebased prevention programs were cognitive-behavioralbased interventions. However, recent prevention interventions focused only on building cognitive-behavioral skills of young people without understanding the sociocultural context of depression. ${ }^{13}$ Spence pointed out that ignorance of environmental factors (i.e., culture and religion) may likely cause the failures of previous universal interventions for depression in adolescents. Hence, all kinds of prevention interventions are now important for a dual approach to build individual skills to reduce risks of depression and also to enhance the environmentally protective factors at the same time.

Recently, a few studies were published on selective and indicated prevention interventions for adolescent depression. Clark et al. found that group cognitive intervention was significantly more efficient than the usual care 
for adolescents who had depressive parents, to prevent depression during a 14-month follow-up period. However, this selective prevention program found that the treatment versus control difference would decrease with time. ${ }^{14}$ Although short-term treatments were unlikely to change any long-term outcome after the discontinuation of treatment, ${ }^{15}$ pharmacological, non-pharmacological, and combined treatments of depression were strongly suggested rather than indicated prevention for youths with prior depressive episodes. ${ }^{16}$

Since there were no reviews that evaluated the long-term effectiveness of psychological intervention to prevent a new onset of depression, supporting evidence is needed to determine specific prevention interventions for recurrent depression in both clinical and community settings.

To date, there are no systematic reviews that focus on psychological intervention in preventing recurrence of depressive episode in adolescents. Therefore, the objective of this study was to systematically review the evidence of psychosocial treatment for recurrence prevention in adolescent depression.

\section{Material and Methods}

\section{Search strategy and study selection}

In June 2015, Embase (1980-2014), Medline (19462014), Global Health (1910-2015), and PsychINFO (20022014) were searched from 1994 to the end of December 2014. The author read all abstracts of the retrieved articles from the search in order to exclude articles which were not eligible.

The search terms were comprised of four components. First, a literature search used the diagnostic categories of depressive disorders in ICD or DSM [“Depression” (“Dysthymia” or “Unipolar Depression”)]to generate the target population. Second, the search narrowed to studies which were undertaken in "Adolescent" ("youth" or "young people") for those age groups in the population.
Third, the databases were searched for articles including words related to recovery prevention programs in depressive disorder. The search included combinations of key words regarding the outcome of "relapse prevention" or "recurrent" as they are overlapping meanings in some articles regarding the definition by the American Academy of Child and Adolescent Psychiatry. Finally, the author searched "psychological intervention" or "psychotherapy" for nonpharmacological interventions to prevent recurrent depression in adolescents.

\section{Further searching}

Various key journals, for instance the Journal of the American Academy of Child and Adolescent Psychiatry, the European Child and Adolescent Psychiatry, and Archives of General Psychiatry, were hand searched by the same search strategy and key words. In addition, key authors (Clarke et al., 1999; Rohde et al., 2006; Stice et al., 2008; Birmaher et al., 2000) ${ }^{11,14,15,17,18}$ were also manually searched for reassurance that relevant articles were not overlooked. This was supplemented by a hand search from the lists of references.

\section{Inclusion criteria}

This systematic review indicated inclusion and exclusion criteria based on the study titled Psychotherapy for depression in children and adolescents: study protocol for a systematic review and network meta-analysis. ${ }^{19}$ Hence, the inclusion criteria were:

-English language

-Original and published journal articles

-Published between 1994 and 2014

-Evidence level $1 \mathrm{~b}-3 \mathrm{~b}^{20}$

-Outcome measurements in participants who already received treatment for depressive disorder

-Participants aged between 10 and 19 years old as this age range is defined as the age of adolescence by WHO (2014) 
-Depressive disorder was clinically screened or recorded regarding International Statistical Classification of Diseases and Related Health Problems 9th/10th or Diagnostic and Statistical Manual of Mental Disorders III/ IV Edition/V Edition Text Revision or depressive status defined as scoring above a certain cut-off on a depression rating scale of standard depressive screening (e.g., the Children's Depression Inventory, Beck Depression Inventory, and Center for Epidemiologic Study Depression Scale)

-Control condition in the articles could be waiting list control (WL), usual care (UC) or (psychotherapy/ pharmacological) placebo. These control conditions were viewed as the independent nodes. ${ }^{19}$

\section{Exclusion criteria}

1. Investigation of children and young people with a known case of physical or other psychiatric diseases as the primary diagnosis (e.g., schizophrenia), because the effectiveness of psychotherapy might be affected by these comorbidities ${ }^{19}$

2. Studies that included psychological intervention combined or compared with pharmacological treatment, such as combining different psychological interventions or psychotherapy and antidepressants, to directly determine the uncontaminated effectiveness of psychological treatment $^{19}$

\section{Data extraction and quality evaluation}

Titles and abstracts of the articles were read by the first author for the purpose of excluding unqualified articles. Then, full-text articles were obtained.

The outcome summarization, within and across the included studies in this review, were categorized as low of risk bias, unclear risk of bias, and high risk of bias. The risk of bias was assessed in each domain of the included studies: sequence generation, allocation concealment, blinding of participants/personnel and outcome assessor, incomplete outcome data, selective outcome reporting, and other sources of risk of bias. In the case of low risk of bias for all domains within a study, all information from the study had to be at low risk of bias. But if one or more domains were unclear or seriously weakened the confidence of the results, the studies were classified as unclear or high risk of bias. ${ }^{22}$

The authors assessed all included studies of each domain and summarized both within and across the studies as low risk of bias, unclear risk of bias or high risk of bias. Disagreements were resolved by both authors.

\section{Results}

The electronic search theoretically found 1,339 relevant titles of studies. The number of excluded articles was 1001: 388 due to duplication and 623 due to their irrelevant grounds (Figure 1). Others were retrieved in full-text for further assessment. Then 6 articles were excluded for particular reasons (Table 1). Therefore, 8 articles were included in the study from the electronic search. The manual search with the same search terms found an additional 4 articles. A total of 12 articles were included in this systematic review.

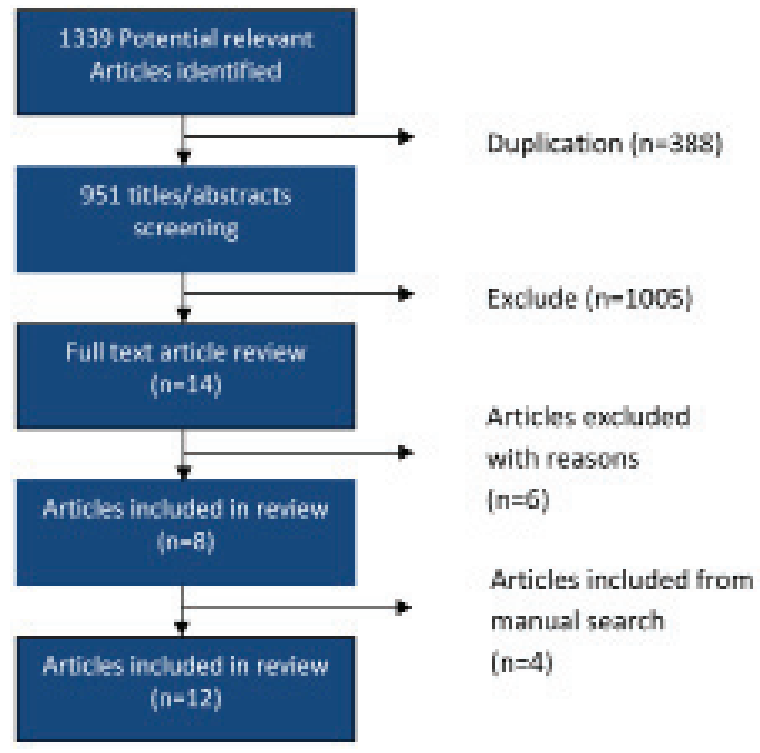

Figure 1 Flow chart of study selection 
Table 1 Studies that were excluded from the analysis

\begin{tabular}{ll}
\hline Study & Reason for exclusion \\
\hline Horowitz et al. $(2006)^{10}$ & Participants did not fully meet clinical criteria of depression \\
Weisz et al. $(2006)^{32}$ & Meta-analysis study \\
Gladstone et al. $(2009)^{33}$ & Systematic review study \\
Dietz et al. $(2014)^{34}$ & No follow-up of recurrence after post-treatment evaluation \\
Zhou et al. $(2014)^{35}$ & Systematic review and open-label studies included \\
Van Zoonen et al. $(2014)^{36}$ & Participants did not fulfill clinical criteria of depression \\
\hline
\end{tabular}

\section{Description of included studies}

Overall, the included studies were divided into two groups based on their primary goals of psychological interventions for adolescent depression. The first group aimed to prevent depression in high-risk youths in a community setting, including participants with a history of prior depression. The other group targeted the nonpharmacological interventions in adolescents who were diagnosed as depressive disorders and had already received some treatments in a clinical setting (Table 2).

The first group or the indicated prevention group included 5 studies: Rohde et al. (2006), ${ }^{18}$ Stice et al. (2008), ${ }^{11}$ Stice et al. (2010), ${ }^{23}$ Beardslee et al. (2013), ${ }^{24}$ and Rohde et al. (2014). ${ }^{25}$ The other group included 7 studies of treatments by Mufson \& Fairbanks (1996), ${ }^{26}$ Renaud et al. (1998), ${ }^{27}$ Birmaher et al. (2000), ${ }^{15}$ Clark et al. (1999), ${ }^{17}$ Sanford et al. (2006), ${ }^{28}$ Trowell et al. (2007), ${ }^{29}$ and Weitkamp et al. $(2014)^{30}$ which were interventions for treatment in clinical settings. Except for the Clark et al. (1999) study, which was a time series (quasi-experimental study/ quasi $\mathrm{RCT}$ ), all of them were designed to be randomized control trial $(\mathrm{RCT})$ studies. $^{17}$

Five of the 12 studies in this systematic review [Clarke et al. (1999), ${ }^{17}$ Rohde et al. (2006), ${ }^{18}$ Stice et al. [2008], ${ }^{11}$ Stice et al. (2010), ${ }^{23}$ and Beardslee et al. (2013) ${ }^{24}$ ] indicated full high quality randomized control trials and a time series with a low risk of bias. They were appropriate designs with sequence generation, randomization, blinding technique, and outcome reports. All studies specified the drop-out rate and their reasons for missing data. However, the 3 studies of Birmaher et al. (2000), ${ }^{15}$ Renaud et al. (1998), ${ }^{27}$ and Rohde et al. (2012) were classified as unclear and with higher risks of bias and the other 4 studies could be categorized as high-risk bias studies since their limitations could significantly alter the results ${ }^{31}$ (Table 3 ).

\section{Participant characteristics}

A total of 1,641 participants were included in this systematic review (1996-2014). Most participants in this systematic review were adolescent females (13-19 years old). All studies were conducted in Europe, Canada, and the USA.

In terms of the exclusion criteria, every study excluded psychosis, eating disorder, bipolar disorders, obsessivecompulsive disorder, physical and sexual abuse, brain disease, substance use, mental retardation, and anxiety disorder. In terms of ethnicity, the authors accepted that the outline of the participant ethnicities in their studies did not represent all ethnicities in the normal population as the main ethnicities in the sample sizes were minorities, as well as participants in the Interpersonal Psychotherapy for Depressed Adolescents (IPT-A) study of Mufson \& Fairbanks (1999) who were all Latinos and African-Americans (Figure 2). 


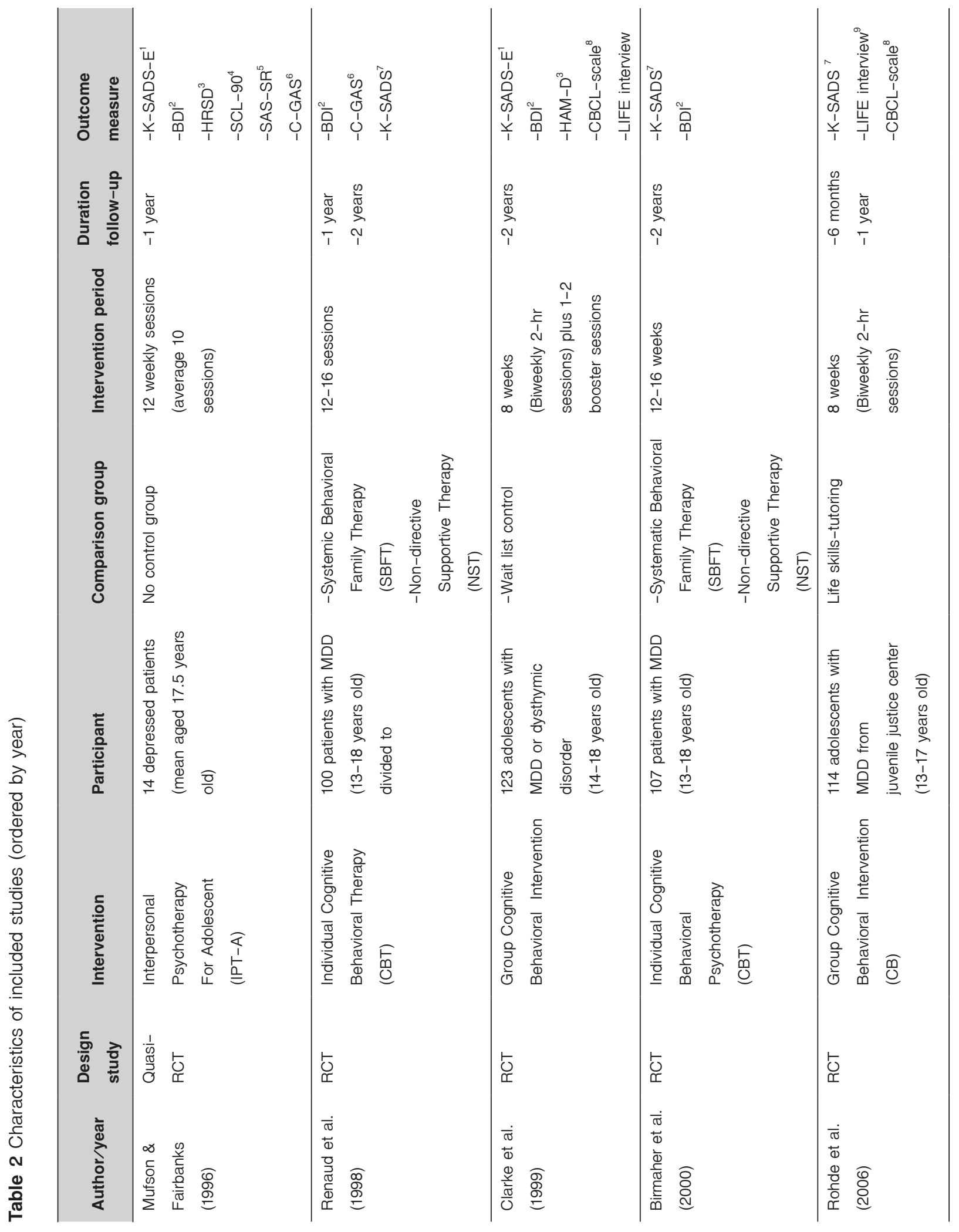




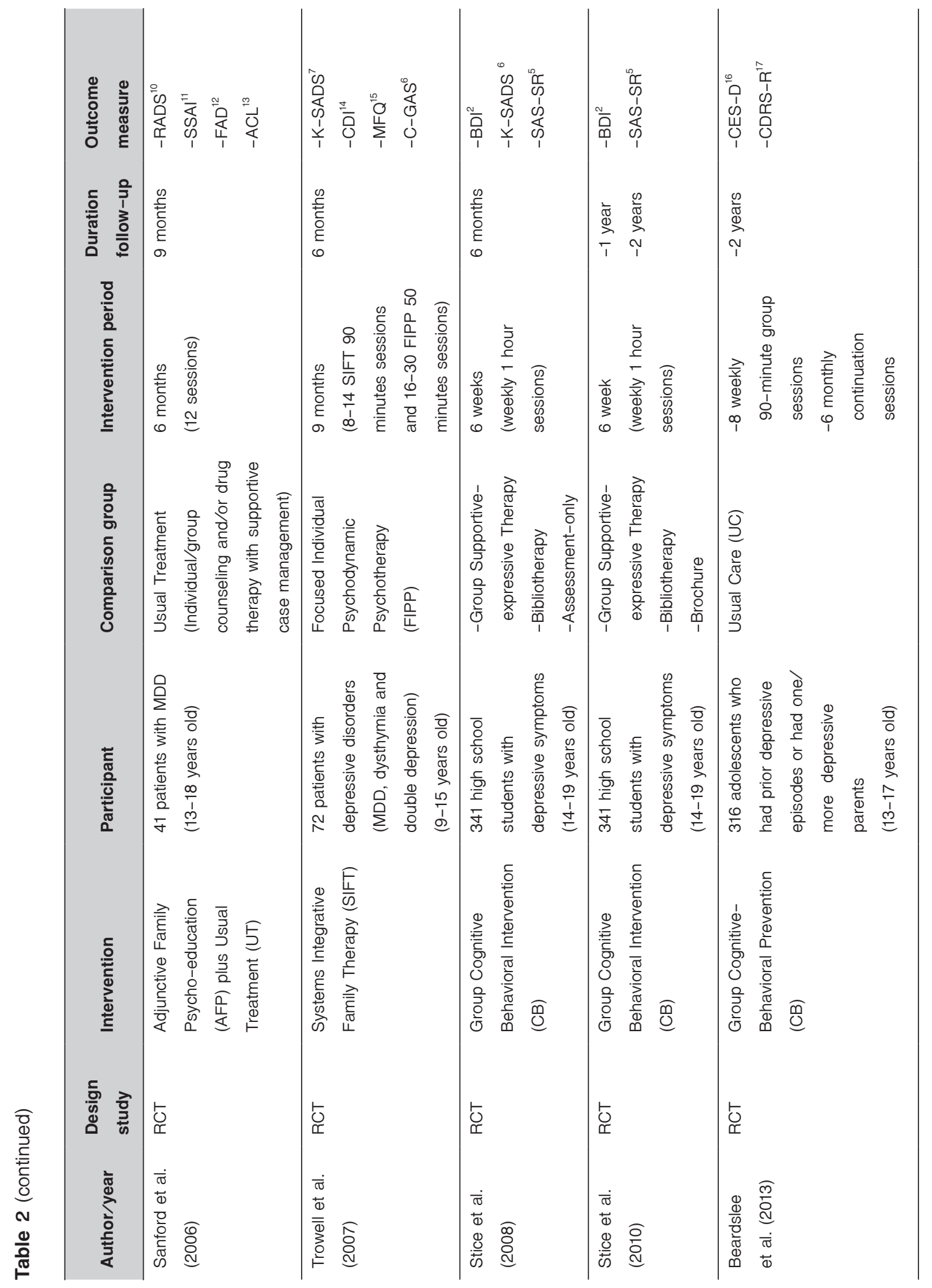




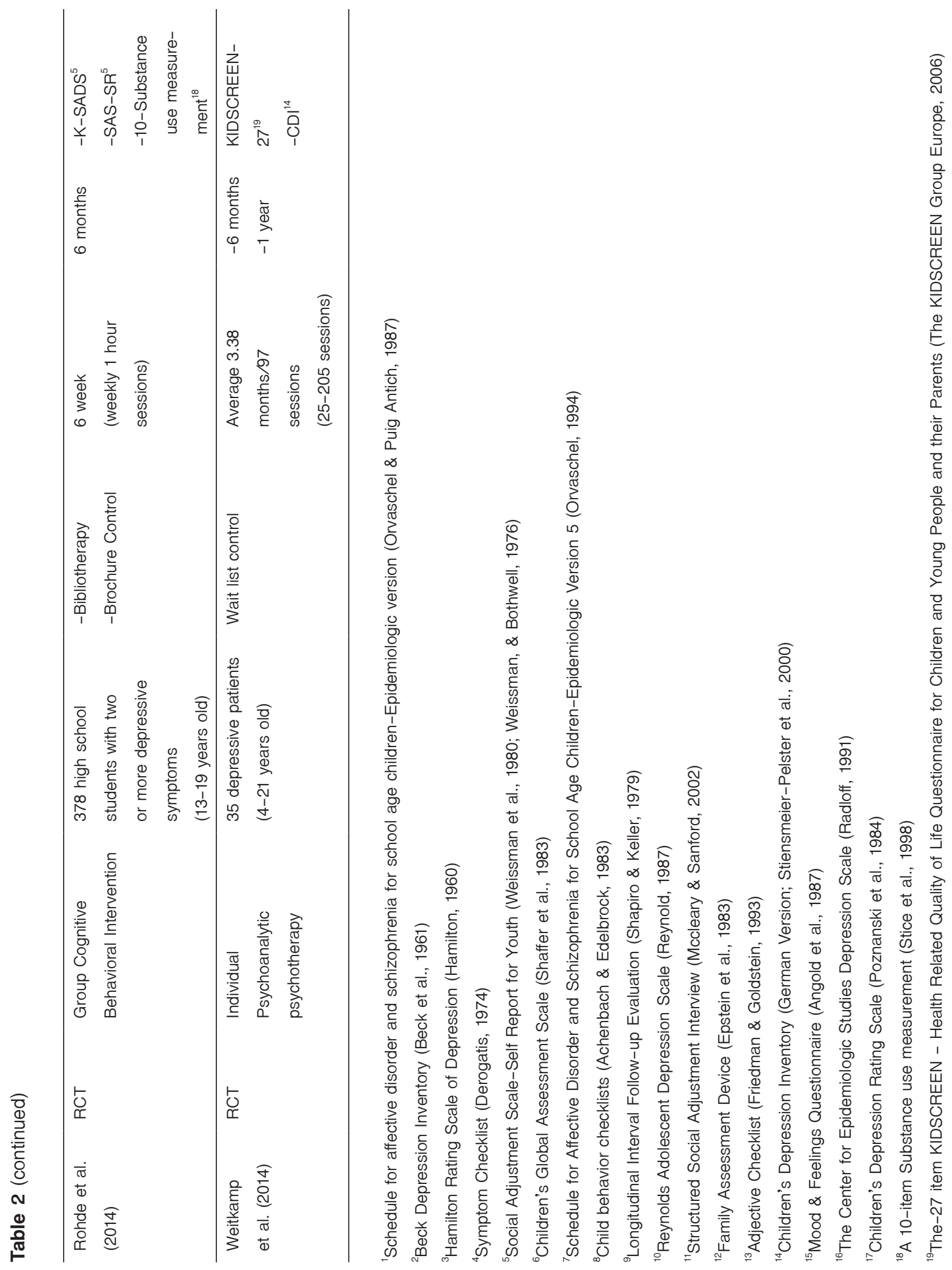


Table 3 Quality assessment: The Cochrane Collaboration's tool to assess the risk of bias (ordered by year)

\begin{tabular}{|c|c|c|c|c|c|c|}
\hline Study & $\begin{array}{l}\text { Sequence } \\
\text { generation }\end{array}$ & $\begin{array}{l}\text { Allocation } \\
\text { concealment }\end{array}$ & $\begin{array}{l}\text { Blinding of } \\
\text { participant and } \\
\text { outcome } \\
\text { (Assessment) }\end{array}$ & $\begin{array}{l}\text { Complete } \\
\text { outcome } \\
\text { data }\end{array}$ & $\begin{array}{l}\text { High-selective } \\
\text { outcome } \\
\text { reporting }\end{array}$ & $\begin{array}{l}\text { Free of } \\
\text { other bias }\end{array}$ \\
\hline Mufson \& Fairbanks (1996) & Low & Low & High & Low & Low & Unclear \\
\hline Renaud et al. (1998) & Low & Low & Low & Unclear & Low & Low \\
\hline Birmaher et al. (2000) & Low & Unclear & Low & Low & Low & Low \\
\hline Clarke et al. (1999) & Low & Low & Low & Low & Low & Low \\
\hline Rohde et al. (2006) & Low & Low & Low & Low & Low & Low \\
\hline Sanford et al. (2006) & Low & Low & High & Low & Low & Unclear \\
\hline Trowell et al. (2007) & Low & Unclear & High & Low & Low & High \\
\hline Stice et al. (2008) & Low & Low & Low & Low & Low & Low \\
\hline Stice et al. (2010) & Low & Low & Low & Low & Low & Low \\
\hline Rohde et al. (2012) & Unclear & Unclear & Low & Unclear & Low & Low \\
\hline Beardslee et al. (2013) & Low & Low & Low & Low & Low & Low \\
\hline Weitkamp et al. (2014) & Unclear & Unclear & High & Low & Low & Low \\
\hline
\end{tabular}

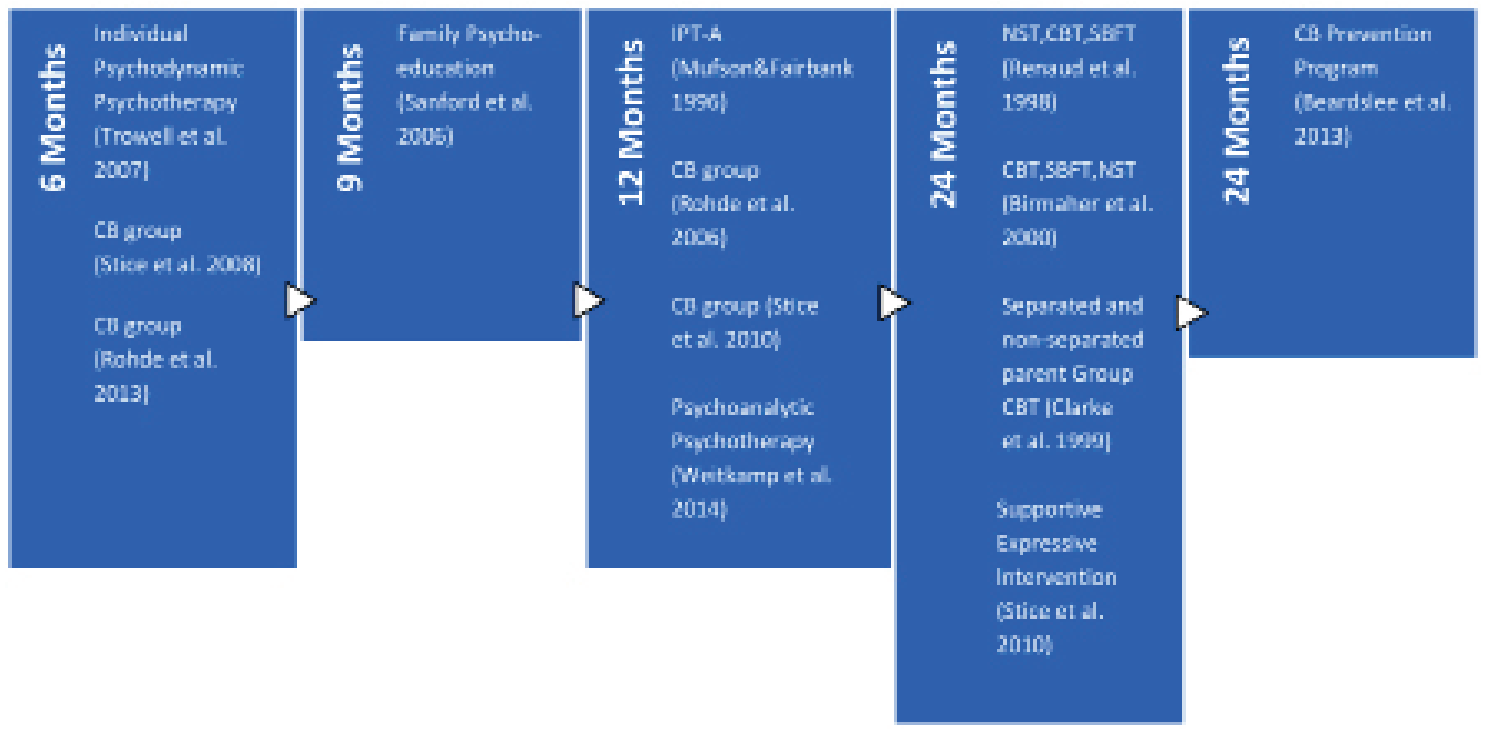

Figure 2 Summary of effective interventions for recurrent prevention (ordered by year and timeline) 


\section{6-month recurrence prevention}

The first study by Stice et al. (2008) showed that brief cognitive behavioral therapy (CBT) intervention produced a significantly greater depressive symptom reduction than the control group and the treatment effect was clinically significant in preventing a new episode of depression for at least 6 months. The effect size of the CBT depression intervention program at 6-month follow-up was medium $(\mathrm{d}=0.42){ }^{11}$

Other interventions that showed persistent effectiveness at 6 months following treatment were individual psychodynamic psychotherapy and family therapy. Trowell et al. (2007) found that both of them could prevent recurrent depression at 6 months of follow-up. Individual psychodynamic psychotherapy appeared to have a more rapid improvement. However, family therapy was more effective than individual psychodynamic psychotherapy in cases of double depression or major depressive disorder on top of dysthymia. ${ }^{29}$

Intervention by Rohde et al. (2014) in another CBT group also appeared to have persistent treatment outcomes preventing depressive onset for more than 6 months. Major depressive disorder (MDD) onset was about two to eight times higher in usual care without psychological intervention compared to group CBT intervention. ${ }^{25}$

\section{9-month recurrence prevention}

At 9 months following intervention, family psychoeducation (FPE) was shown to decrease depressive symptoms in the study by Sanford et al. (2006). The effects were medium (Standardized $E S=0.64$ ). However, the small sample size $(n=41)$ of this study brought about low accuracy in the estimated treatment effect. $^{28}$

\section{2-month recurrence prevention}

The results of the study by Mufson \& Fairbanks (1999) suggested that the 12-week interpersonal psycho- therapy (IPT-A) for depressed adolescents could maintain clinical improvement for 12 months following completion of the intervention. Although the outcome should be interpreted with caution according to the small sample size $(\mathrm{n}=14)$ and attrition rate, this study reported that IPT-A resulted in fewer depressive symptoms, improved social function, and less co-morbidity than pre-intervention. ${ }^{26}$

A 12-month recovery of MDD was also reported in a study of group CBT by using an intent-to-treat analytic approach. $^{18}$ The participants reported the onset of recovery by 12 -week post-intake $(43.0 \%)$ and $77.0 \%$ had recovered at the end of the 12-month follow-up period.

Supportive-expressive intervention in the Stice et al. (2010) study was reported to marginally reduce depressive symptoms at 12-month follow-up. However, this therapeutic effect did not significantly persist at 24 months of followup. $^{23}$

Finally, Weitkamp et al. (2014) showed a sustained therapeutic effect of individual psychoanalytic psychotherapy up to 1 year after treatment completion. Psychoanalytic therapy showed significantly reduced depressive symptoms and increased quality of life, compared with the wait-list control in the range of a moderate effect size by the end of the 12-month follow-up period $(E S=4.36){ }^{30}$

\section{4-month recurrence prevention}

Renaud et al. (1998) discovered no differences in the rates of recovery and MDD onset at 24-month follow-up among CBT, systematic behavioral family therapy (SBFT), and non-directive supportive therapy (NST). However, different population groups showed various therapeutic effects. In patients who had rapid response to treatment, NST showed a greater proportion of improved functioning $(87.5 \%)$ than the non-rapid response group (54.5\%). ${ }^{27}$

Similarly, Birmaher et al. (2000) found that CBT did not confer longer-term therapeutic effects over family and supportive therapy regarding recurrence prevention 
in depressive adolescents. Across 3 psychotherapy groups, $92.0 \%$ of the patients recovered after 24 months following psychotherapy trials. ${ }^{15}$

Nevertheless, Clarke et al. (1999) reported that both the immediate and 24-month follow-up therapeutic effects of group CBT was superior to a wait-list control $(E S=0.61)$. Parental involvement in group modality was not associated with clinical improvement. ${ }^{17}$ Another study by Stice et al. (2010) also found that group CBT was an effective intervention that reduced depressive symptoms up to 24 months following treatment and the effect size was medium $(E S=0.30){ }^{23}$

\section{3-month recurrence prevention}

Beardslee et al. (2013) conducted the longest duration of assessment in the included studies. They stated that the cognitive behavioral prevention (CBP) program showed a significant therapeutic effect compared with usual care (UC) over the 33 months of follow-up (number needed to treat of 10 at $95 \%$ confidence interval=5-2624). Although the later onset rate of depression was slower following completion of the CBP program, the average onset of depression between CBP and UC showed no difference in cases of ongoing parental depression (number needed to treat of 54$).{ }^{24}$

\section{Discussion}

The main objective of this systematic review was to evaluate the evidence of psychological interventions in depressive adolescents to prevent recurrent depression. Twelve studies involving a total of 1,641 participants were included in this review. There were different effects of psychosocial interventions for depression following treatment completion that relied on follow-up durations.

The results from twelve studies suggested that cognitive behavioral group interventions (Clarke et al., $1999 ;{ }^{17}$ Stice et al., 2008; ${ }^{11}$ Stice et al., 2010; ${ }^{23}$ Rohde et al., 2006; ${ }^{18}$ Rohde et al., 2014; ${ }^{25}$ Beardslee et al., 2013 ${ }^{24}$ ) showed the greatest effects on recurrence prevention since these studies claimed that CBT groups could prevent recurrence of depression from 6 to 33 months. Even though individual CBT interventions were also effective in preventing recurrent depression up to 24 months following treatment completion, ${ }^{15,27}$ there were no differences among the individual CBT, non-directive supportive psychotherapy (NST) or systematic behavioral family therapy (SBFT).

Family interventions represented their effectiveness on recurrence prevention up to 9 months in cases of adjunctive family psycho-education (FPE) ${ }^{28}$ and up to 24 months for SBFT. ${ }^{15,27}$ Although IPT-A could prevent recurrent depression for 12 months following treatment, the study bias and small sample size may raise some doubts on the results and their recommendations. ${ }^{26}$ Likewise, psychoanalytic psychotherapy and individual psychodynamic psychotherapy were also doubtful in their outcomes of 6 and 12 month-recurrence prevention due to their small sample size and higher risks of bias. ${ }^{29}$

Even though this systematic review showed outcomes that suggest support for group CBT, it is too early to conclude that group CBT is the best intervention for recurrence prevention of depression in adolescents. This is because there were few studies that showed the effectiveness of other psychosocial interventions, and there was a lack of data regarding recurrence prophylaxis which is hard to differentiate from treatment continuation. In addition, the effectiveness of group CBT has never been compared with other psychological interventions (e.g., IPT-A, psychodynamic psychotherapy) for recurrence prevention. To fill the gap of knowledge, it is necessary to determine which intervention could prevent a new onset of depressive episode in adolescents.

However, grey literature and unpublished resources were not searched and counted in this systematic review. Also, non-English articles were not included as English I 
anguage only was one of the inclusion criteria. This limitation may lead to overlooking articles conducted in non-western countries.

\section{Conclusion}

The finding of one quasi-randomized control trial and eleven randomized control trials indicated that group cognitive behavioral interventions had the benefit of preventing recurrent depression in depressive adolescents. Other psychological interventions for recurrent depression which were suggested less frequently were family intervention (systematic behavioral family therapy and adjunctive family psycho-education) and psychodynamic/psychoanalytic psychotherapy. However, there are particular risks of bias and limitations in some populations that may result in outcomes that are more tentative.

\section{Acknowledgement}

This study is a dissertation in part fulfillment of the requirements for a Master of Science in Children and Adolescent Mental Health and Psychological Practice, University of Edinburgh. The first author has been fully granted her master's degree by the Faculty of Medicine, Prince of Songkla University, Thailand. The authors wish to thank Glenn Shingledecker for his assistance with the English proofreading of this manuscript.

\section{References}

1. World Health Organization. Young people: health risks and solutions (Fact sheet No. 345) [homepage on the Internet]. Geneva: WHO; 2011 [cited 2015 May 12]. Available from: http://www.who.int/mediacentre/factsheets/fs345/en/ index.html

2. Merry S, Spence S. Attempting to prevent depression in youth: a systematic review of the evidence. Early Interv Psychiatry 2007;1:128-37.

3. Bitsika V, Sharpley C. Comorbidity of anxiety-depression among Australian university students: implications for student counsellors. Br J Guid Counc 2012;40:385-94.
4. Fergusson D, Boden J, Horwood L. Recurrence of major depression in adolescence and early adulthood, and later mental health, educational and economic outcomes. Br J Psychiatry 2007;191:335-42.

5. Rao U, Ryan N, Birmaher B, Dahl R, Williumsom D, Kaufman J, et al. Unipolar depression in adolescents: clinical outcome in adulthood. Am Acad Child Adolesc Psychiatry 1995;34:56678.

6. Calear A, Christensen H. Systematic review of school-based prevention and early intervention programs for depression. $J$ Adolesc 2010;33:429-38.

7. Katon W, Rutter C, Ludman E, Von Korff M, Lin E, Simon G, et al. A randomized trial of relapse prevention of depression in primary care. Arch Gen Psychiatry 2001;58:241-7.

8. Merikangas KR, He JP, Burstein M, Swanson SA, Avenevoli S, Cui L, et al. Lifetime prevalence of mental disorders in U.S. adolescents: results from the National Comorbidity Survey Replication-Adolescent Supplement (NCS-A). J Am Acad Child Adolesc Psychiatry 2010;49:980-9.

9. Sato S, Ishikawa S, Togasaki Y, Ogata A, Sato Y. Long-term effects of a universal prevention program for depression in children: a 3-year follow-up study. Child Adolesc Ment Health 2012;18:103-8.

10. Horowitz J, Garber J. The prevention of depressive symptoms in children and adolescents: a meta-analytic review. J Consult Clin Psychol 2006;74:401-15.

11. Stice E, Rohde P, Seeley J, Gau J. Brief cognitive-behavioral depression prevention program for high-risk adolescents outperforms two alternative interventions: a randomized efficacy trial. J Consult Clin Psychol 2008;76:595-606.

12. Ishikawa S, Iwanaga M, Yamashita B, Sato H, Sato S. Longterm effects of social skills training on depressive symptoms in children. Jpn J Educ Psychol 2010;58:372-84.

13. Herman K, Reinke W, Parkin J, Traylor K, Agarwal G. Childhood depression: rethinking the role of the school. Psychol Sch 2009;46:433-46.

14. Clarke G, Hornbrook M, Lynch F, Polen M, Gale J, Beardslee $\mathrm{W}$, et al. A randomized trial of a group cognitive intervention for preventing depression in adolescent offspring of depressed parents. Arch Gen Psychiatry 2001;58:1127-34.

15. Birmaher B, Brent D, Kolko D, Baugher M, Bridge J, Holder D, et al. Clinical outcome after short-term psychotherapy for 
adolescents with major depressive disorder. Arch Gen Psychiatry 2000;57:29-36.

16. Ryan N. Treatment of depression in children and adolescents. Lancet 2005;366:933-40.

17. Clarke G, Rohde P, Lewinsohn P, Hops H, Seeley J. Cognitivebehavioral treatment of adolescent depression: efficacy of acute group treatment and booster sessions. J Am Acad Child Adolesc Psychiatry 1999;38:272-9.

18. Rohde P, Seeley J, Kaufman N, Clarke G, Stice E. Predicting time to recovery among depressed adolescents treated in two psychosocial group interventions. J Consult Clin Psychol 2006;74:80-8.

19. Qin B, Zhou X, Michael K, Liu Y, Whittington C, Cohen D, et al. Psychotherapy for depression in children and adolescents: study protocol for a systematic review and network metaanalysis. BMJ Open 2015;5:1-7.

20. Liesegang TJ. Evidence-based medicine: principles for applying the users' guides to patient care. Guyatt GH, Haynes RB, Jaeschke RZ, Cook DJ, Green L, Naylor CD, Wilson MC, Richardson WS for the Evidence-Based Medicine Working Group. JAMA 2000;284:1290-6. Am J Ophthalmol 2001;131: 153.

21. Guyatt G, Haynes R, Jaeschke R, Cook D, Green L, Naylor C, et al. Users' guides to the medical literature. JAMA 2000;284: 1290-6.

22. Cochrane Oral Health. Resource [homepage on the Internet]. Manchester: University of Manchester [cited 2015 May 13]. Available from: http://oralhealth.cochrane.org/resources

23. Stice E, Rohde P, Gau J, Wade E. Efficacy trial of a brief cognitive-behavioral depression prevention program for highrisk adolescents: effects at 1- and 2-year follow-up. J Consult Clin Psychol 2010;78:856-67.

24. Beardslee W, Brent D, Weersing V, Clarke G, Porta G, Hollon $\mathrm{S}$, et al. Prevention of depression in at-risk adolescents. JAMA Psychiatry 2013;70:1161-70.

25. Rohde P, Stice E, Shaw H, Briere F. Indicated cognitive behavioral group depression prevention compared to bibliotherapy and brochure control: acute effects of an effectiveness trial with adolescents. J Consult Clin Psychol 2014;82:65-74.

26. Mufson L, Fairbanks J. Interpersonal psychotherapy for depressed adolescents: a one-year naturalistic follow-up study. J Am Acad Child Adolesc Psychiatry 1996;35:1145-55.

27. Renaud J, Brent D, Baugher M, Birmaher B, Kolko D, Bridge J. Rapid response to psychosocial treatment for adolescent depression: a two-year follow-up. J Am Acad Child Adolesc Psychiatry 1998;37:1184-90.

28. Sanford M, Boyle M, Mccleary L, Miller J, Steele M, Duku E, et al. A pilot study of adjunctive family psychoeducation in adolescent major depression: feasibility and treatment effect. Am Acad Child Adolesc Psychiatry 2006;45:386-495.

29. Trowell J, Joffe I, Campbell J, Clemente C, Almqvist F, Soininen $\mathrm{M}$, et al. Childhood depression: a place for psychotherapy. Eur Child Adolesc Psychiatry 2007;16:157-67.

30. Weitkamp K, Daniels J, Hofmann H, Timmermann H, Romer G, Wiegand-Grefe S. Psychoanalytic psychotherapy for children and adolescents with severe depressive psychopathology: preliminary results of an effectiveness trial. Psychotherapy 2014;51:138-47.

31. Rohde P, Stice E, Gau J, Marti C. Reduced substance use as a secondary benefit of an indicated cognitive-behavioral adolescent depression prevention program. Psychol Addict Behav 2012;26:599-608.

32. Weisz JR, McCarty CA, Valeri SM. Effects of psychotherapy for depression in children and adolescents: a metaanalysis. Psychol Bull 2006;132:132-49.

33. Gladstone T, Beardslee W. The prevention of depression in children and adolescents: a review. Can J Psychiatry 2009;54: 212-21.

34. Dietz L, Marshal M, Burton C, Bridge J, Birmaher B, Kolko D, et al. Social problem solving among depressed adolescents is enhanced by structured psychotherapies. J Consult Clin Psychol 2014;82:202-11.

35. Zhou X, Michael K, Liu Y, Del Giovane C, Qin B, Cohen D, et al. Systematic review of management for treatment-resistant depression in adolescents. BMC Psychiatry [serial on the Internet]. 2014 Nov [cited 2015 Jul]; 14(1). Available from: https://www.ncbi.nlm.nih.gov/pmc/articles/PMC4254264/

36. Van Zoonen K, Buntrock C, Ebert D, Smit F, Reynolds C, Beekman A, et al. Preventing the onset of major depressive disorder: a meta-analytic review of psychological interventions. Int J Epidemiol 2014;43:318-29. 